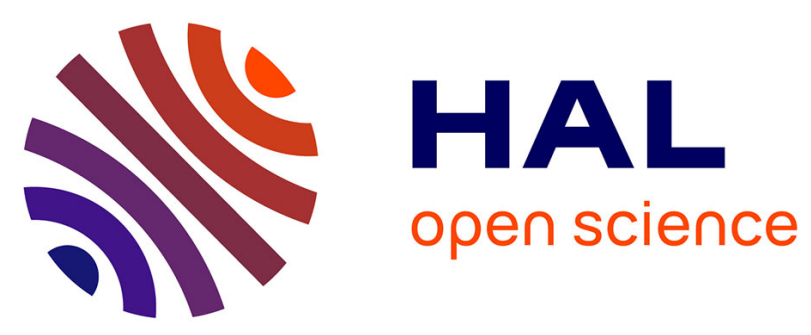

\title{
Intercomparison of Permittivity Measurement Techniques for Ferroelectric Thin Layers
}

Patrick Queffelec, Vincent Laur, Alexis Chevalier, Jean-Michel Le Floch, Damien Passerieux, Dominique Cros, Valérie Madrangeas, Arnaud Le Febvrier, Stéphanie Députier, Maryline Guilloux-Viry, et al.

\section{To cite this version:}

Patrick Queffelec, Vincent Laur, Alexis Chevalier, Jean-Michel Le Floch, Damien Passerieux, et al.. Intercomparison of Permittivity Measurement Techniques for Ferroelectric Thin Layers. Journal of Applied Physics, 2014, 115 (2), pp.024103. 10.1063/1.4858388 . hal-00929884

\section{HAL Id: hal-00929884 https://hal.science/hal-00929884}

Submitted on 17 Jun 2016

HAL is a multi-disciplinary open access archive for the deposit and dissemination of scientific research documents, whether they are published or not. The documents may come from teaching and research institutions in France or abroad, or from public or private research centers.
L'archive ouverte pluridisciplinaire HAL, est destinée au dépôt et à la diffusion de documents scientifiques de niveau recherche, publiés ou non, émanant des établissements d'enseignement et de recherche français ou étrangers, des laboratoires publics ou privés. 


\title{
Intercomparison of permittivity measurement techniques for ferroelectric thin layers
}

\author{
P. Queffelec, ${ }^{1}$ V. Laur, ${ }^{1}$ A. Chevalier, ${ }^{1}$ J.-M. Le Floch, ${ }^{2}$ D. Passerieux, ${ }^{3}$ D. Cros, ${ }^{3}$ \\ V. Madrangeas, ${ }^{3}$ A. Le Febvrier, ${ }^{4}$ S. Députier, ${ }^{4}$ M. Guilloux-Viry, ${ }^{4}$ G. Houzet, ${ }^{5}$ T. Lacrevaz, ${ }^{5}$ \\ C. Bermond ${ }^{5}$ and B. Fléchet ${ }^{5}$ \\ ${ }^{1}$ Lab-STICC, UMR CNRS 6285, Université de Bretagne Occidentale, 6 av. Le Gorgeu, CS 93837, \\ 29238 Brest Cedex, France \\ ${ }^{2}$ School of Physics, The University of Western Australia, 35 Stirling Hwy, Crawley, Western Australia 6009, \\ Australia \\ ${ }^{3}$ XLIM, UMR CNRS 6172, Université de Limoges, 123 av. A. Thomas, 87060 Limoges Cedex, France \\ ${ }^{4}$ ISCR, UMR CNRS 6226, Université de Rennes 1, 35042 Rennes Cedex, France \\ ${ }^{5}$ IMEP-LAHC, UMR CNRS 5130, Université de Savoie, 73376 Le Bourget du Lac, France
}

(Received 24 September 2013; accepted 10 December 2013; published online 13 January 2014)

\begin{abstract}
The dielectric properties of a $\mathrm{KTa}_{0.65} \mathrm{Nb}_{0.35} \mathrm{O}_{3}$ ferroelectric composition for a submicronic thin layer were measured in the microwave domain using different electromagnetic characterization methods. Complementary experimental techniques (broadband methods versus resonant techniques, waveguide versus transmission line) and complementary data processing procedures (quasi-static theoretical approaches versus full-wave analysis) were selected to investigate the best way to characterize ferroelectric thin films. The measured data obtained from the cylindrical resonant cavity method, the experimental method that showed the least sources of uncertainty, were taken as reference values for comparisons with results obtained using broadband techniques. The error analysis on the methods used is discussed with regard to the respective domains of validity for each method; this enabled us to identify the best experimental approach for obtaining an accurate determination of the microwave dielectric properties of ferroelectric thin layers. (C) 2014 AIP Publishing LLC. [http://dx.doi.org/10.1063/1.4858388]
\end{abstract}

\section{INTRODUCTION}

Electromagnetic (EM) characterization of materials is a prerequisite step before their use in microwave applications: radar, mobile phones, wireless communications, etc. It enables circuit designers to precisely determine of the EM properties (permittivity, permeability, and electric conductivity) of the materials composing studied devices or the systems. It also allows quality control of materials in the manufacturing process and validation of models of interaction between EM waves and matter by comparison with experiments. It is widely recognized that poor design of microwave devices is often caused by erroneous values of the EM properties of the circuit substrate.

Many measurement techniques have been developed over recent years to determine the EM properties of materials. These are based on the measurement of the perturbation of propagating or standing waves (e.g., coaxial line-based reflection/transmission method, cavity perturbation technique, etc.). Commercialization of high-performance vector network analyzers (VNA) has led to a gradual increase in the automation of these techniques.

Today, the characterization of homogeneous and isotropic dielectric thick samples is conducted relatively well using conventional methods with transmission lines or resonant cavities, combined with suitable data processing programs. However, this is not the case for materials that exhibit an unusual shape, such as thin films and painted layers; in such cases, obtaining an accurate measurement is still problematic. Difficulties are increased when studying the influence of external parameters (such as a mechanical stress, shift in temperature, or DC biasing field) on the EM properties of these materials.

Ferroelectric thin films fall into this category of materials. Their submicron thickness introduces the problem of the sensitivity of the measurement method used. The study of variations in their dielectric properties under the action of a static electric field requires specific techniques with command electrodes. Finally, the influence of the metallizations, caused by possible inter-diffusion, and of the type of substrate chosen for thin layer deposition (mono- or polycrystalline), must also be examined.

Solving the problem of how to perform such an analysis is paramount because these materials are needed to fit the specifications of new applications in different domains. Thin films are considered very promising materials for improving RF circuit performances through new functionalities: tunability and miniaturization of filters, phase shifters, antennas, etc.

In this context, the French laboratories XLIM, IMEPLAHC, and Lab-STICC, who are specialized in the EM characterization of materials, decided to conduct an intercomparison of permittivity measurements for ferroelectric thin films. The strategy adopted was to use different characterization techniques to measure the complex permittivity of a single ferroelectric composition $\mathrm{KTa}_{0.65} \mathrm{Nb}_{0.35} \mathrm{O}_{3}$ (KTN) deposited on different substrates at the ISCR laboratory. Even though the selected methods do not necessarily operate at the same frequencies because of their own domain of validity, all experimental data collected were obtained in the 
centimeter wave range $(3-30 \mathrm{GHz})$ of the microwave domain. Each laboratory involved in the intercomparison procedure conducted its own characterization without knowing the results obtained by the other partners. The potential differences in temperature and humidity encountered in different laboratories during the measurements were not taken into account in this intercomparison experiment.

\section{EM CHARACTERIZATION OF DIELECTRIC THIN FILMS}

Permittivity is a physical quantity that describes the ability of a medium to become polarized (appearance of dipolar moments) under the action of an electric field. In the dynamic regime, in order to account for dissipative effects in the matter, $\varepsilon$ is represented by a complex quantity $\left(\varepsilon=\varepsilon^{\prime}-\mathrm{j} \varepsilon^{\prime \prime}\right)$ that depends on the field frequency (dispersion), the homogeneity of the medium, its possible anisotropic character and external parameters such as temperature and mechanical stresses. ${ }^{1}$ In the case of ferroelectric media, permittivity varies if the polarization state of the material is changed by the application of a static electric field: a property used to ensure the tunability of microwave functions (filters, phase shifters, etc.). ${ }^{2-7}$ This offers an interesting alternative to the more conventional technological solution based on the use of active elements (PIN diodes, varactors) and, more recently, micro-electromechanical systems (MEMS).

The EM characterization of a ferroelectric thin layer consists in determining, at one frequency or over a wide range, its relative permittivity compared with a vacuum $\varepsilon_{r}=\varepsilon / \varepsilon_{0}$ and its loss tangent $\tan \delta=\varepsilon^{\prime \prime} / \varepsilon^{\prime}$. Two operating procedures exist to measure these two physical quantities in the microwave domain. Mono-frequency methods use resonating modes in cavities. ${ }^{8-13}$ The insertion of a sample into the cavity induces a shift in its resonant frequency and quality factor. The measurement of these variations enables highly accurate determination of the quantities $\varepsilon_{r}$ and $\tan \delta$ at a $\sin -$ gle frequency. Even if mono-frequency methods give very accurate results, broadband methods can also be used in the microwave spectrum since they allow the dispersion of EM properties of the constructed ferroelectric materials to be studied. To determine dielectric properties of materials over a wide range of frequencies, reflection/transmission measurement techniques, based on the use of rectangular waveguides or transmission lines such as the coaxial type, were developed in the late 20th century. ${ }^{14-16}$ These techniques have been successfully applied to characterize dielectric or magnetic homogeneous, isotropic materials in bulk form (thick samples). Intercomparison of measurements, carried out using the two major operating procedures (mono-frequency ${ }^{17}$ and broadband ${ }^{18}$ ), accompanied by an analysis of the different error sources affecting the measurements, made it possible to definite the best experimental protocols and implementation of the most robust experimental data processing programs to ensure an accurate EM characterization of bulk materials. In this field, the reflection/transmission technique is based on the standard use of a coaxial line. ${ }^{19}$ Unfortunately, this regular method, which consists in inserting the sample to be characterized (torus of a few millimeters thick) into the line, is poorly adapted for the characterization of thin films. Alternative solutions in planar technologies (microstrip or coplanar) started to appear in the 1990s to work around this inadequacy, whilst retaining the advantage of the broad operating band of transmission lines. ${ }^{20-22}$ The philosophy of this approach is to choose measurement cells that guarantee a strong interaction between the EM wave and the thin film, which is made possible by a favorable direction and concentration of the EM field with regard to the planar geometry of the material.

For ferroelectric thin films, the approach consists in integrating the layer, and its substrate, directly into the transmission line. ${ }^{23}$ The metallic layers (strip and ground plane) are deposited on the thin layer, which is itself deposited on its own substrate. The measurement of the line propagation constant makes it possible to determine the complex relative permittivity of the material to be characterized. ${ }^{24}$ Coplanar waveguide (CPW) structures seem to have the best suited topology for ferroelectric thin films because they ensure a tangential direction of the electric field compared with the plane of the layer, which is a required condition for good method sensitivity. CPW technology also allows easy integration of a static electrical command to study the DC field-dependent permittivity of the material. Another advantage of this solution relates to the in-situ character of the conducted measurement, because the permittivity of the layer is determined in a very similar configuration to those used in practice in $\mathrm{CPW}$ microwave devices. This is particularly important in the case of an anisotropic medium for which the values of the inplane and out-of-plane permittivities are different.

However, broadband CPW methods are destructive, and the parameter determined from the measured scattering parameters (S-parameters) of the line corresponds in reality to the effective permittivity of the line and not that of the thin layer, which is only a part (with a very small volume) of the overall structure. To retrieve the complex relative permittivity of the thin layer, a final step must be implemented in order to reverse the electromagnetic analysis of the CPW structure. However, as the thin layer is integrated under the metallic layers of the CPW structure, it is impossible to perform a differential measure that would eliminate cell imperfections (metallic losses, radiation, dielectric losses of the thick substrate) from the measured S-parameters. Many error sources therefore affect the accuracy of the results. These are caused by uncertainties during the data processing procedure in certain geometric parameters (thickness of the thin layer or metallic layers) and electromagnetic parameters (permittivity of the substrate layer, electrical conductivity of the metallic layers ${ }^{25}$ or pollution that the latter can cause by interdiffusion, radiation, or dispersion) of the line. In some cases, these different elements may finally have a greater influence on the measured parameters (S-parameters) than that generated by the ferroelectric thin film, which displays a very small thickness (typically, a few hundred nanometers).

The objective of the present intercomparison measurements is to identify the potential of different EM characterization methods for the accurate determination of the dielectric properties of ferroelectric thin layers. From these comparisons performed on a same ferroelectric thin film, 
analysis of the obtained results should allow us, first, to define an experimental protocol and a data processing procedure for each method, to improve the accuracy of the results obtained and, ultimately, to define an EM characterization standard for dielectric thin films based either on a single method or on a combination of complementary methods in terms of accuracy and bandwidth.

\section{KTN FERROELECTRIC THIN FILMS: SYNTHESIS AND MICROSTRUCTURAL PROPERTIES}

Previous reports have investigated dielectric properties of $\mathrm{KTa}_{0.6} \mathrm{Nb}_{0.4} \mathrm{O}_{3}$ thin films on polycrystalline alumina substrates in the microwave frequency range, ${ }^{26,27}$ but comparisons of the measured permittivity values would be risky because deposition processes differed: pulsed laser deposition ${ }^{26}$ versus chemical solution deposition. ${ }^{27}$ KTN thin films were deposited on various substrates suitable for the microwave range, especially $\mathrm{LaAlO}_{3}$ and $\mathrm{MgO} .^{28}$ In this study, $400 \mathrm{~nm}$ thick KTN films were deposited by pulsed laser ablation (KrF laser, $\lambda=248 \mathrm{~nm}$ ) at $700^{\circ} \mathrm{C}$ under 0.3 mbar oxygen pressure. This KTN composition was selected for its Curie temperature, $T_{c}$, which is slightly lower than room temperature (on bulk material $\mathrm{T}_{\mathrm{c}}=269 \mathrm{~K}^{29}$ ), in order to favour a high tunability, while avoiding hysteretic effects at room temperature. On both $\mathrm{LaAlO}_{3}$ and $\mathrm{MgO}$ substrates, the films exhibited a (100) epitaxial growth, as evidenced by X-Ray Diffraction performed in $\theta-2 \theta, \omega$-, and $\varphi$-scan modes. ${ }^{28}$ The structural quality appears to be higher on $\mathrm{LaAlO}_{3}$ on which the full widths at half maximum, $\Delta \omega$ and $\Delta \varphi$, of the $\omega$-scan (rocking-curve) and $\varphi$-scan are generally lower than on $\mathrm{MgO}^{28} \mathrm{On}$ $\mathrm{MgO}$, the presence of a secondary minor (110) orientation is commonly observed, as shown by Fig. 1. The Scanning Electron Microscopy (SEM) micrographs are in agreement with the XRD measurements, where (100) epitaxial thin films were revealed (in-plane ordering of plate square grains) on both substrates, in addition to the coexistence of the (110) secondary orientation on $\mathrm{MgO}$ substrate illustrated by the elongated grains) (Fig. 2). Despite the difference in crystalline quality, a KTN sample deposited on $\mathrm{MgO}$ was next chosen for the inter-comparison of microwave measurements. The main reason for this choice is the lower permittivity of $\mathrm{MgO}$ $\left(\varepsilon_{\mathrm{r}} \sim 9-10\right)$, which is isotropic, whereas the presence of twins in $\mathrm{LaAlO}_{3}$ can induce modifications in its permittivity, which is higher $\left(\varepsilon_{\mathrm{r}} \sim 24\right)$ than that of $\mathrm{MgO}$. A value that is too high for the substrate permittivity would induce a decrease of the sensitivity of the measurement method used for the EM characterization of the ferroelectric layer.

\section{DESCRIPTION AND PRINCIPLES OF MICROWAVE CHARACTERIZATION METHODS}

This section will briefly present the experimental principle of the EM characterization methods used by the different laboratories in this intercomparison.

\section{A. Rectangular waveguide method}

The first method, developed at Lab-STICC, is based on the measurement of the scattering parameters (S-parameters)
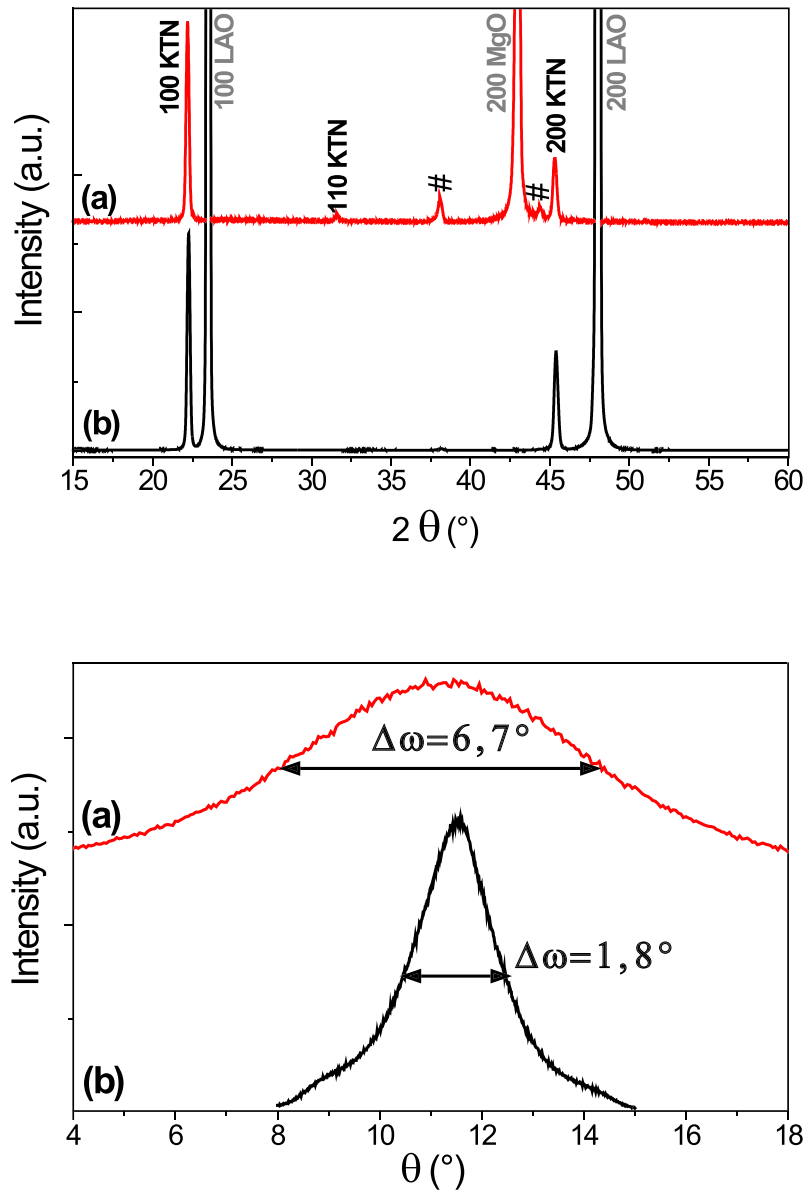

FIG. 1. $\theta-2 \theta$ XRD patterns of the KTN (65/35) grown on (100) $\mathrm{MgO}$ (a) and (100) $\mathrm{LaAlO}_{3}$ (b) substrates. \# indicates diffraction peaks of the sample holder. The (100) orientation of $\mathrm{LaAlO}_{3}$ refers to the pseudo cubic lattice.

of a rectangular waveguide loaded by the ferroelectric thin film deposited on its own substrate. ${ }^{30}$ As illustrated in Fig. 3, the square-shaped bilayer sample under study is set on its thickness in the mid-plane of the cross-section of an X-band $(8-12 \mathrm{GHz})$ rectangular waveguide. A TRL calibration procedure enabled us to correct systematic errors and to shift the reference planes closer to the sample to be characterized.

Dynamic analysis of the measurement cell (modematching method) consists in evaluating the electric- and magnetic-field components that are solutions of the Maxwell equations; this enables the calculation of the direct problem and determination of the theoretical S-parameters of the loaded waveguide. This approach allowed us to take into
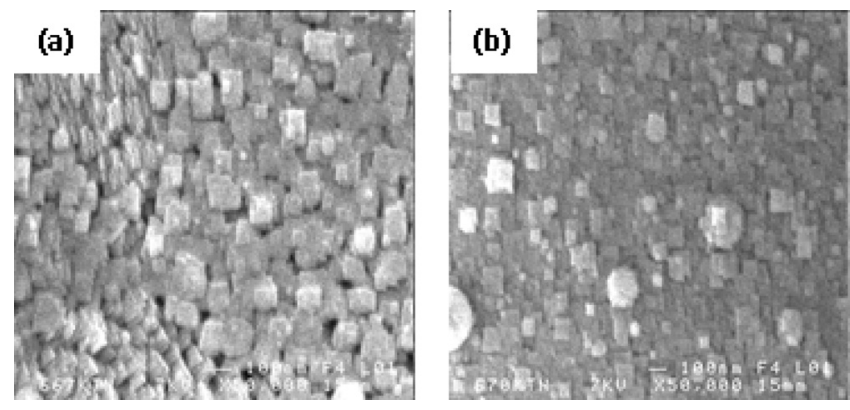

FIG. 2. SEM micrographs of the KTN thin films deposited on (a) $\mathrm{MgO}$ and (b) $\mathrm{LaAlO}_{3}$ 


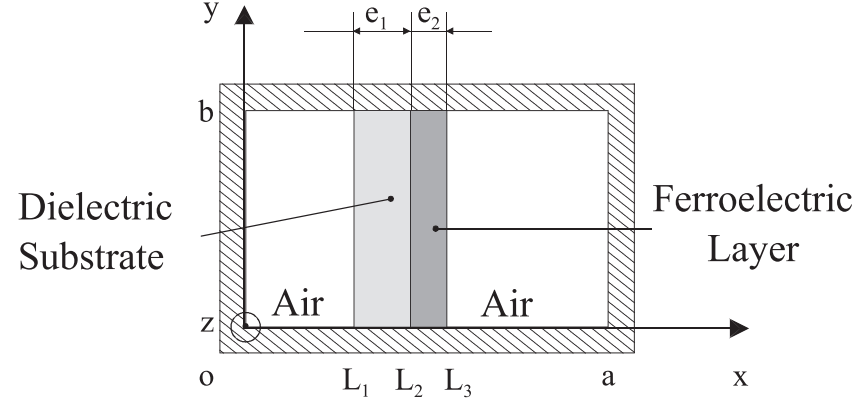

FIG. 3. Cross-section of the rectangular waveguide partially filled with a thin ferroelectric layer deposited on its substrate.

account not only the dominant mode $\left(\mathrm{TE}_{10}\right)$, but also the higher order modes that can be excited at the discontinuities in the waveguide. ${ }^{31}$

The waveguide cross-section was separated into 4 layers (air/dielectric/thin film/air). The dispersion relationships for each mode were established. A mode-matching analysis was then used to take into account the abrupt discontinuities between the empty waveguide regions and the one partly filled with the ferroelectric layer being characterized. Finally, the complex dielectric constant was extracted by minimizing the quadratic error between the measured Sparameters and those issued from the direct problem.

Fig. 4(a) shows the permittivity spectrum of a KTN thin film deposited on $\mathrm{MgO}$ substrate, extracted using the rectangular waveguide technique in the $8-12 \mathrm{GHz}$ frequency band.

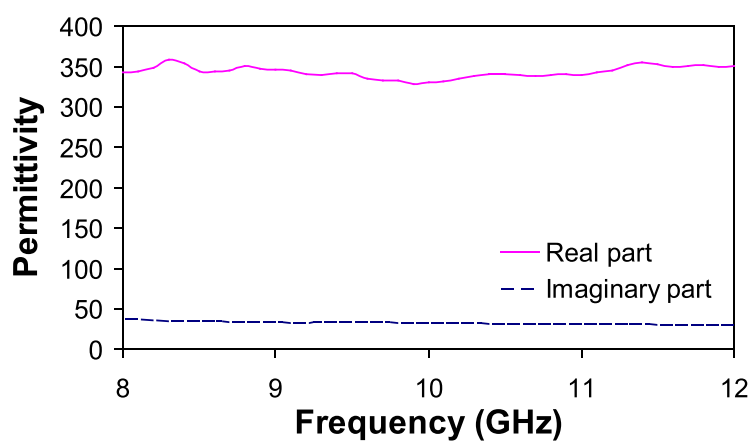

(a)

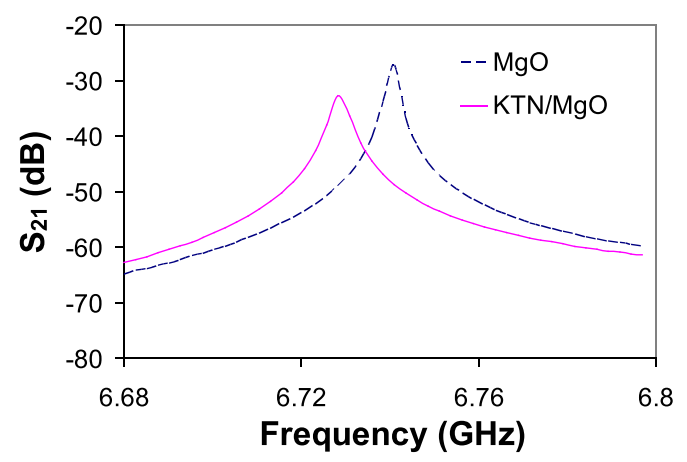

(b)

FIG. 4. (a) Rectangular waveguide method: Permittivity spectra of a KTN thin film deposited on $\mathrm{MgO}$ substrate and (b) Cavity perturbation method: Transmission coefficients (modulus) of the cavity loaded with an $\mathrm{MgO}$ substrate and with a KTN/MgO bilayer.
The dielectric properties of the ferroelectric film appear to be non-dispersive in this band, with mean values of 343 and 32.6 for the real and imaginary parts of the permittivity, respectively.

\section{B. Cavity perturbation method}

The second method, developed at Lab-STICC, enabled us to extract the dielectric properties of the ferroelectric thin film from the variations in resonance frequency and the quality factor of a rectangular cavity induced by the insertion of the material under test. The resonant cavity consists of a 5$\mathrm{cm}$ long X-band rectangular waveguide section excited by circular apertures. For this structure, the resonant modes are of $\mathrm{TE}_{10 \delta}$ type. When the integer number $\delta$ is odd, the electric field shows a maximum magnitude at the centre of the cavity. The sample to be characterized was placed in this region to increase the sensitivity of the method. A slot, situated at the centre of the cavity, enabled insertion of the sample.

Two steps were then required to characterize the ferroelectric thin film. First, an $\mathrm{MgO}$ substrate was inserted into the cavity. The resonance frequency and the quality factor of the cavity were calculated and were considered as reference values. Second, a KTN/MgO sample was inserted into the cavity. At this step, the $\mathrm{MgO}$ substrate must present the same dimensions and dielectric properties as the one considered in the first step. Thus, the dielectric constant and losses of the KTN thin film modify the resonance frequency and quality factor of the cavity. As variations of these parameters are low, the complex permittivity of the ferroelectric thin film can be calculated analytically using a low perturbation approach. ${ }^{32,33}$

In our case, the cavity loaded with the $\mathrm{MgO}$ substrate showed a resonance frequency of $6.7403 \mathrm{GHz}$ and a quality factor of 3768 for the $\mathrm{TE}_{101}$ mode. When a KTN/MgO sample was inserted into the cavity, the resonance frequency shifted to $6.7287 \mathrm{GHz}$ and the quality factor decreased to 1839 (Fig. 4(b)). The low perturbation approach then enabled the dielectric properties of the thin film to be extracted: $\varepsilon^{\prime}=312$ and $\tan \delta=8 \times 10^{-2}$.

\section{Resonant method using a cylindrical cavity and dielectric resonators}

XLIM developed a resonant system at $12 \mathrm{GHz}$, made up of a cylindrical cavity associated with two identical dielectric resonators placed face to face inside the cavity (Fig. 5). ${ }^{34}$ The variations of resonance frequency and the quality factor of the $\mathrm{TE}_{01 \delta}$ mode induced by the insertion of a sample (substrate, thin film + substrate) between the dielectric resonators allows its permittivity and loss tangent to be extracted using simulation software based on the method of lines (MOL).

The dielectric properties extracted using this method are extremely sensitive to the accuracy of information on the thicknesses of the different materials, which is notably difficult to measure accurately in ferroelectric thin films. New methods are currently being sought to minimize this measurement uncertainty. Another major limitation of this method concerns the permittivity-thickness product, which 

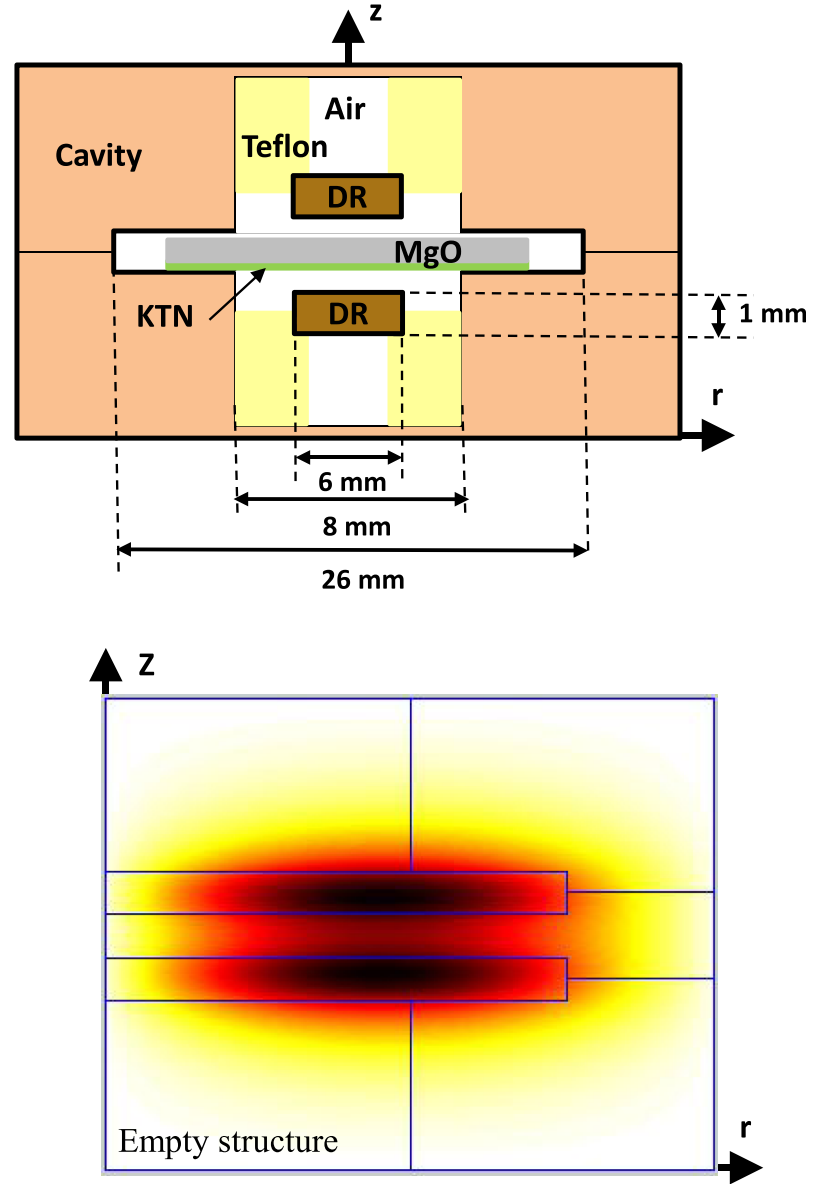

FIG. 5. Measurement cell and electric field in the structure for the three measurement steps.

must be sufficiently significant to induce noticeable changes between the calibration (substrate) and measurement steps (thin film + substrate). A rigorous EM analysis is then needed to extract the dielectric properties of the thin film. Our analysis was based on the 2.5D MOL, which allowed us to take into account very thin dielectric layers and avoid meshing problems associated with 3D methods. The complexity of the structure required that a successive approach be used for the extraction of the dielectric properties, whose convergence is ensured by the Newton-Raphson method.

\section{Broadband characterization using a CPW structure}

A broadband characterization method $(40 \mathrm{MHz}-67 \mathrm{GHz})$ based on the measurement of the lineic electric parameters (capacity $C$ and conductance $G$ ) of coplanar transmission lines (CPW) was used at IMEP-LAHC. Following this method, the KTN layer to be tested was first deposited on an $\mathrm{MgO}$ substrate, then metallic electrodes were deposited on the KTN before chemical engraving (Fig. 6). Metallization dimensions were chosen by making a compromise between maximizing the interaction between the electric propagated field and the KTN layer, and minimizing conduction losses in the CPW line. S-parameters of the CPW line were measured using an Agilent network analyzer (PNA-XN5247A) calibrated by an LRRM procedure and treated to obtain the values of $C$ and $G$. Extraction of the

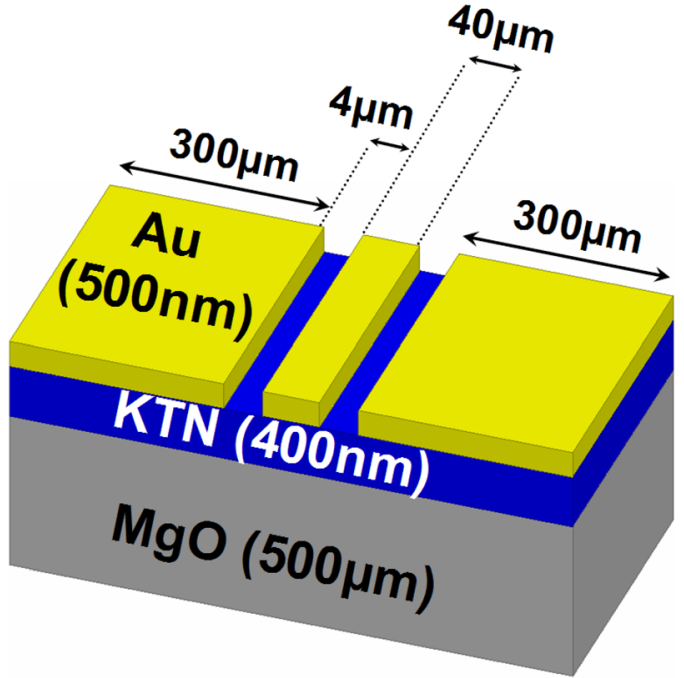

FIG. 6. Cross-section of the CPW structure.

complex permittivity of the KTN thin film was then performed through an iterative optimization process based on Powell-Simplex algorithms. ${ }^{35}$ Real and imaginary parts of the estimated thin film permittivity were adjusted simultaneously until the values of $C$ and $G$ simulated by the EM solver HF2D fit the measured values. ${ }^{36}$ To do this, a specific error function, strongly dependent on the permittivity value and defined in a two-dimensional space $\left(\varepsilon^{\prime}, \varepsilon^{\prime \prime}\right)$, was minimized. This extraction process is used at each frequency and offers the benefit of covering a very wide range of frequencies. This reveals a distributed relaxation of the KTN permittivity, which is well described by a Cole-Davidson model. Fig. 7(a) shows the good agreement between measured and calculated data. The choice of this model is well supported in the highest part of the frequency band by the pronounced decrease of the real part and the increase of the imaginary part of the permittivity.

The coplanar lines deposited on KTN and characterized in IMEP-LAHC were also measured at Lab-STICC to retrieve complex permittivity using a different data processing program. Here, the theoretical analysis based on quasistatic approximation consists in using only the transmission

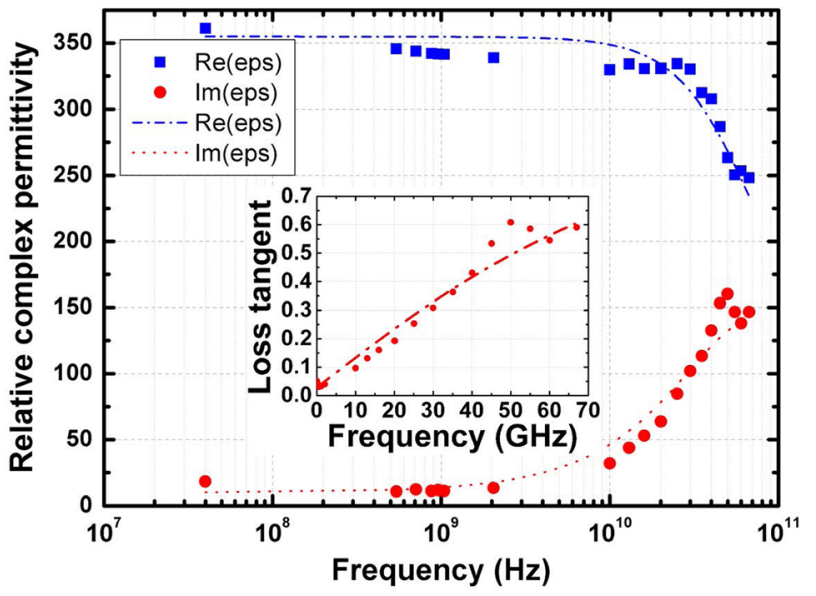

FIG. 7. Measured (symbol) and calculated (line) complex permittivity spectra of the KTN thin film. Insert: dielectric loss tangent. 
parameter $S_{21}$ in the case of non-magnetic materials. The permittivity of the thin film was calculated from the measured effective permittivity of the structure. Using the conformal mapping technique, we obtained analytical expressions that related the complex permittivity of the thin film to the geometrical parameters of the line and the dielectric properties of the substrate. ${ }^{37}$ These analytical relationships enabled us to avoid using numerical optimization procedures to retrieve thin film permittivity. In this method, the different types of losses in the CPW structure were also taken into account: we considered the substrate dielectric, conductor, and radiation losses. ${ }^{25}$

The CPW lines were measured using the Cascade Microtech $^{\circledR}$ probe station and Agilent E8364 VNA in the frequency range [5 $\mathrm{GHz}-50 \mathrm{GHz}$. During the first stage, the device was calibrated using an LRRM procedure. We then performed a differential measurement on two lines of different lengths in order to minimize contact inaccuracy. The measured complex permittivity and loss tangent are shown in Fig. 7.

\section{COMPARISON AND DISCUSSION OF EXPERIMENTAL RESULTS}

The choice of characterization methods for this intercomparison was dictated by their complementarities. First of all, we wanted to compare the suitability of the two usual operating modes for the characterization of thin films; on the one hand, resonant techniques (cavities, resonators) and, on the other hand, the reflection/transmission methods (transmission line, waveguide). For broadband techniques, the interest of our study lies in the comparison between a differential approach (correction of S-parameters by taking into account the measurement of the empty cell) and an overall measurement in CPW line, in which the thin film is integrated. It would be also interesting to study the influence of the theoretical models used for the EM analysis of the different measurement cells on the accuracy of the measured data.

\section{A. Influence of the models used for the EM analysis of the cell}

The first broadband approach, based on the use of a rectangular waveguide, can be considered as non-invasive because of the absence of metallization onto the ferroelectric film, in contrast to the second, for which the CPW metallic layers were directly deposited on the thin-film. As discussed in Sec. II, the latter method does not allow removal of cell imperfections in the absence of a sample (empty cell). In these conditions, the only alternative for the data processing procedure is to accurately predict all the physical phenomena inherent in the measurement cell: skin depth effect in the metallic layers (central strip conductor and ground planes), radiation, dispersion of the EM properties of the substrate, etc. The problem with this approach is the validity of the theoretical model used for the EM analysis of the CPW line. The model must thoroughly describes the skin depth effect in metallic layers without being limited by the high contrast between the respective thicknesses of the different layers present in the structure: a few hundred nanometers for the ferroelectric thin film and a few hundred micrometers for the substrate. To bypass this theoretical difficulty, IMEP-LAHC chose to exploit the lineic parameter capacity $C$ and conductance $G$ of the CPW line, which are not strictly dependent on conductor loss. The modeling of these two quantities was done using an EM solver, which ensures very good calculation accuracy. The difficulty related to the choice of the model for EM analysis of the measurement cell can be illustrated by a comparison of the loss tangent spectra retrieved from the two different data processing programs used with the CPW line. In the [5 GHz-50 GHz] frequency band, the quasi-static approach gives a variation in loss tangent from 0.12 up to 0.25 (Fig. 8(b)), whereas the full-wave analysis predicts variation from 0.04 up to 0.6 (Fig. 7(b)). Since the full-wave analysis enabled us to determine measurable parameters that were independent of conductor loss (capacity $C$ and conductance $G$ ), we can conclude from this comparison that the loss models related to the quasi-static approach do not allow accurate values to be retrieved for the dielectric loss of the ferroelectric thin film. The real parts of the permittivity spectra obtained from the two different programs used to process the CPW measured data were no longer in very good agreement in the upper part of the exploited frequency range (around 33\% relative discrepancy at $50 \mathrm{GHz}$, see Figs. 7 and 8).

For the rectangular waveguide method (see Sec. IV A), a full-wave EM analysis was also necessary to guarantee high accuracy of the calculated S-parameters. In addition to the
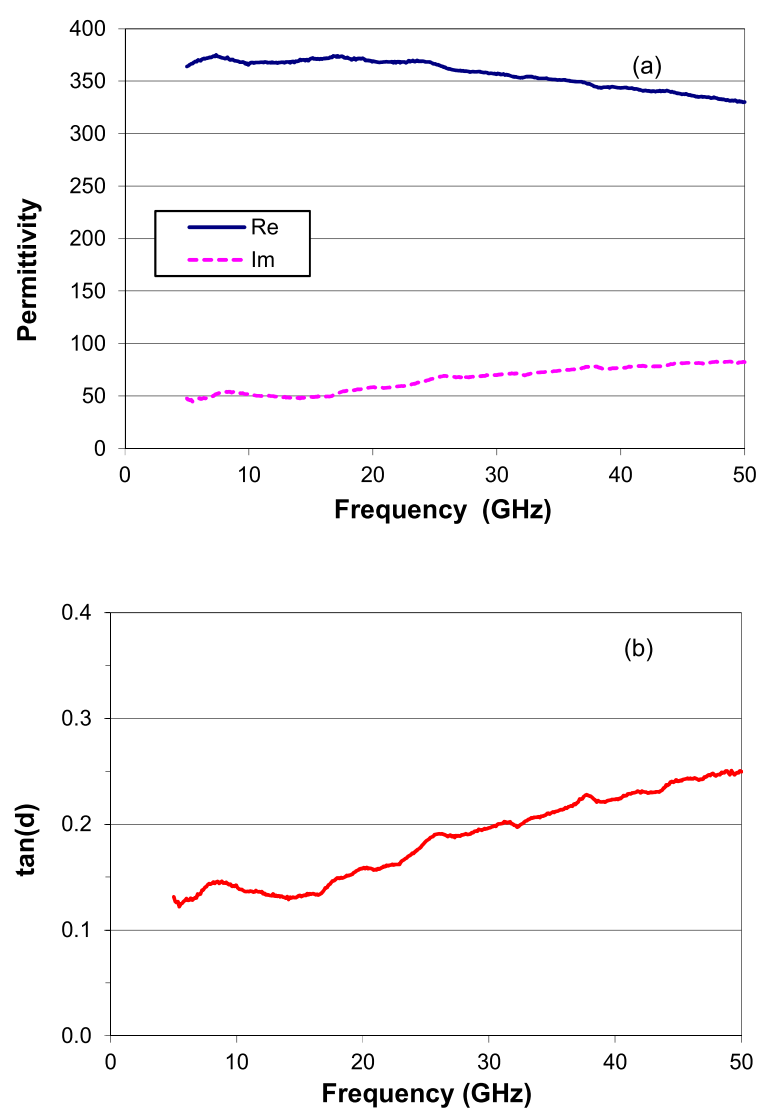

FIG. 8. CPW structure-based characterization of KTN thin film deposited on $\mathrm{MgO}$ : (a) complex permittivity spectra, (b) dielectric loss tangent. 
$\mathrm{TE}_{10}$ dominant mode, six higher-order modes were taken into account in the EM analysis to ensure convergence of the calculations. It is not necessary to have a very accurate description of the skin effect in the metallic walls of the waveguide because of the differential character of the measurement procedure (calibration of the S-parameters by removing the empty cell measurement). Since the data processing programs used to retrieve the permittivity of the ferroelectric thin films from the rectangular waveguide and the CPW line measurements were both based on a full-wave analysis, it is interesting to compare the corresponding results. When comparing the permittivity spectra in Figs. 4(a) and 7(a), we observed a very good agreement between the measured data obtained from both broadband methods based on full-wave analysis. At $10 \mathrm{GHz}$, the real part of the permittivity is equal to 340 and the loss tangent is equal to 0.09 for both methods. These data are in very good agreement with those obtained from the resonant method based on a full-wave analysis $\left(\varepsilon_{r}{ }^{\prime}=352, \tan \delta=0.11\right.$ at $\left.12 \mathrm{GHz}\right)$. In contrast, the low-perturbation approach used with the rectangular cavity predicted a lower value for the real part of the permittivity $\left(\varepsilon_{r}^{\prime}=312\right.$ at $\left.6.7 \mathrm{GHz}\right)$.

From these comparisons, we can conclude that it is preferable to use full-wave analysis in the description of the microwave behavior of measurement cells as this guarantees a higher accuracy of measurements of complex permittivity of ferroelectric thin films.

\section{B. Error analysis}

Results from the measurement campaign on KTN thin films are summarized in Table I. Despite the opposing characters of the operating modes (resonant versus Reflection/transmission techniques) and, in the case of broadband methods, despite the different propagation structures (waveguide versus transmission line) and the different systematic error correction procedures (differential measurement versus global measurement), there is good agreement between dielectric properties retrieved from full-wave analysis-based data processing programs.

The resonant method that uses a cylindrical cavity offers the least sources of uncertainty. This method is based on a differential-type correction of systematic errors, in which the quality factor and the resonance frequency of the empty cavity constitutes the reference. Cavity modeling is rigorous, and the existence of an air gap is not an obstacle. Moreover, measured parameters are less sensitive to random errors (VNA signal to noise ratio) than broadband techniques. However, permittivity is determined at a specified frequency, so an assessment of the dispersion of dielectric properties in ferroelectric thin films is not possible. The main source of uncertainty is related to inaccuracies in the determination of layer thickness due to its inhomogeneity. For the KTN ferroelectric layer characterized during these comparison measurements, it should be noted that the Pulsed Laser Deposition technique used for its construction ensures a relative good homogeneity of layer thickness over the whole surface. The mean relative variation observed for the thickness of submicronic ferroelectric thin films deposited by PLD is around $15 \%^{38}$ over the whole tested surface. This level of inaccuracy leads to a relative error of $12 \%$ for the retrieved permittivity (real part) of the layer. This is confirmed by a previous experimental study, ${ }^{13}$ which showed that it is possible to measure real permittivity of ferroelectric films having submicronic thickness and permittivity range of 100-10000 with uncertainties similar to uncertainties about their thickness. Finally, in this campaign, permittivity measured in the cavity can be considered as the reference value fixed here at $12 \mathrm{GHz}$.

A resonant method based on a rectangular cavity was also tested. Using this approach, permittivity was calculated by the approximation of small perturbations. Even though the resonant frequencies of the two cavities were not identical $(12 \mathrm{GHz}$ and $6.73 \mathrm{GHz})$, their relative proximity led to a difference of $11 \%$ for the real part of the permittivity and $28 \%$ for the loss tangent. This comparison made it possible to estimate the uncertainties brought about by using the weak perturbation assumption to predict the EM field pattern variations in resonant cavities when inserting a dielectric sample.

The two broadband methods led to comparable values of permittivity, close to the reference value obtained from the cylindrical cavity-based method: $\varepsilon_{r}=352$; $\tan \delta=1.12 \times 10^{-1}$. Compared to these reference values, the differences observed at $12 \mathrm{GHz}$ between the waveguide-based method and the method using the CPW structure were $2.5 \%$ and $6.2 \%$, respectively, for the real part of the permittivity and $15 \%$ and $10 \%$ for the loss tangent.

The error source analysis related to the rectangular waveguide-based broadband method showed that the main uncertainty in the measured complex permittivity is due to

TABLE I. Results overview of KTN $(500 \mathrm{~nm})$ thin film deposited on $\mathrm{MgO}(500 \mu \mathrm{m})$ substrate.

\begin{tabular}{|c|c|c|c|c|c|c|}
\hline EM structure & Destructive character & EM analysis of the cell & Operating mode & Frequencies (GHz) & $\varepsilon_{\mathrm{r}}$ & $\tan \delta$ \\
\hline Rectangular waveguide ${ }^{(a)}$ & Non invasive & Full-wave & Broadband & $8-12$ & 343 & $9.5 \times 10^{-2}$ \\
\hline Rectangular Cavity $^{(a)}$ & Non invasive & Quasi-static & Mono-frequency & 6.73 & 312 & $8.0 \times 10^{-2}$ \\
\hline Resonant System $\left(\right.$ cavity + DR) ${ }^{(b)}$ & Non invasive & Full-wave & Mono-frequency & 12 & 352 & $1.12 \times 10^{-1}$ \\
\hline $\mathrm{CPW}$ line $\mathrm{e}^{(\mathrm{c})}$ & Invasive & Full-wave & Broadband & $0.04-67$ & $\begin{array}{c}341(1 \mathrm{GHz}) \\
248(67 \mathrm{GHz})\end{array}$ & $\begin{array}{l}0.033(1 \mathrm{GHz}) \\
0.59(67 \mathrm{GHz})\end{array}$ \\
\hline CPW line ${ }^{(a)}$ & Invasive & Quasi-static & Broadband & $5-50$ & $\begin{array}{c}365(5 \mathrm{GHz}) \\
330(50 \mathrm{GHz})\end{array}$ & $\begin{array}{c}0.12(5 \mathrm{GHz}) \\
0.25(50 \mathrm{GHz})\end{array}$ \\
\hline
\end{tabular}

\footnotetext{
(a) Lab-STICC

(b) XLIM

(c) IMEP-LAHC
} 
imprecision on the permittivity value of the substrate of the thin film. ${ }^{30}$ This error occurred in all broadband methods used for this campaign. However, its influence can vary according to the measurement cell and how the EM wave interacts with the substrate. For example, the CPW structure minimizes this influence because the EM energy is strongly concentrated between the central conductor and the ground planes, around the interface separating the air and the ferroelectric thin film.

Existence of air gap between the bilayered sample and the walls of the waveguide can lead to an underestimation of the real part of the permittivity. Particular attention must be given during the machining of samples in order to minimize uncertainties due to the air gap. This does not occur with the CPW-based method. An error analysis performed during the use of the rectangular waveguide method ${ }^{30}$ enabled us to estimate the influence of an air gap on the accuracy of the measured permittivity. We predicted a relative uncertainty of $10 \%$ for the real part of the permittivity (value around 350) for a $10-\mu \mathrm{m}$ thick air gap. The imaginary part of the permittivity is slightly sensitive to air gaps. A one-centimetre length slot could be machined in the median plane of the rectangular waveguide to facilitate the insertion of the sample to be characterized. This new way of inserting the sample could also make it possible to eliminate the error caused by air gaps.

\section{CONCLUSION}

The unique character of ferroelectric thin films, which results from their submicron thicknesses and related high permittivity values and moderate losses, makes their microwave characterization difficult. This difficulty is increased by the impossibility of separating the thin layer from its substrate. The large difference between the thicknesses of substrate and thin layer requires an accurate determination of the latter's EM properties. Fortunately, this geometric contrast is balanced by a difference in permittivity values in favor of the thin layer.

The first lesson that should be drawn from these intercomparison permittivity measurements performed on a KTN thin layer, is the need to choose thorough theoretical approaches for the EM analysis of the measurement cells. The full-wave analysis (mode-matching method, finite elements method, etc.), which takes into account the physical phenomena inherent to the structures in which the thin layer is inserted (or directly integrated) for characterization, must be consistently favored over quasi-static theories (weak perturbation approximation, quasi-TEM assumption, etc.). This conclusion is logical since the contribution of the thin layer to the measured S-parameters is always in the minority compared with the contributions of the other elements, particularly the metallic layers and substrate.

The most surprising conclusion drawn from the results obtained in this study is that the differences observed between the loss tangent determined from broadband methods and that given by the resonant technique are relatively low. The greater sensitivity of the broadband methods to the signal-to-noise ratio could suggest a far greater difference than that actually observed. This good result demonstrates the relevance of the two broadband methods tested for the EM characterization of ferroelectric thin layers. The waveguide-based method could be defined as a standard for EM characterization of dielectric thin films as long as the data processing program and related experimental protocol allow: (1) elimination of the air gaps (or correction according to their influence) and, (2) use of the measurement performed on the substrate of the thin layer as a reference in the differential approach. The CPW-based method is essential for in-situ characterization over a broad range of frequencies. It also offers the advantage of allowing the study of the variation of ferroelectric layer permittivity under the action of a static field.

\section{ACKNOWLEDGMENTS}

Scanning electron microscopy observations were performed at CMEBA in the University of Rennes 1, France.

\footnotetext{
${ }^{1}$ A. R. V. Hippel, Dielectric Materials and Applications (MIT Press, Cambridge, MA, 1954).
}

${ }^{2}$ R. R. Romanofsky, F. W. Van Keuls, and F. A. Miranda, J. Phys. IV 8(3), 171-194 (1998).

${ }^{3}$ J. Nath, W. M. Fathelbab, P. G. Lam, D. Ghosh, S. Aygün, K. G. Gard, J. P. Maria, A. I. Kingon, and M. B. Steer, IEEE MTT-S Int. Microw. Symp. Dig. 2006, 552-555.

${ }^{4}$ S. Courrèges, Y. Li, Z. Zhao, K. Choi, A. T. Hunt, and J. Papapolymerou, IEEE Trans. Microwave Theory Tech. 57(12), 2872-2881 (2009).

${ }^{5}$ G. Vélu, K. Blary, L. Burgnies, J. C. Carru, E. Delos, A. Marteau, and D. Lippens, IEEE Microw. Wirel. Compon. Lett. 16(2), 87-89 (2006).

${ }^{6}$ D. Kim, Y. Choi, M. Ahn, M. G. Allen, J. S. Kenney, and P. Marry, IEEE Microw. Wirel. Compon. Lett. 13(10), 434-436 (2003).

${ }^{7}$ Q. Simon, Y. Corredores, X. Castel, R. Benzerga, R. Sauleau, K. Mahdjoubi, A. Le Febvrier, S. Députier, M. Guilloux-Viry, L. Zhang, P. Laurent, and G. Tanne, Appl. Phys. Lett. 99, 092904 (2011).

${ }^{8}$ B. W. Hakki and E. D. Coleman, IEEE Trans. Microwave Theory Tech. 8, 402-410 (1960).

${ }^{9}$ W. E. Courtney, IEEE Trans. Microwave Theory Tech. 18, 467-485 (1970).

${ }^{10}$ R. J. Cook, Microwave Cavity Methods (IPC Science and Technology Press, 1973), pp. 12-27.

${ }^{11}$ J. Krupka and A. Kedzior, Electron Technol. 14, 67-79 (1981).

${ }^{12}$ R. N. Clarke and C. B. Rosenberg, J. Phys. E: Sci. Instrum. 15(9), 9-24 (1982).

${ }^{13}$ J. Krupka, W.-T. Huang, and M.-J. Tung, Ferroelectrics 335, 89-94 (2006).

${ }^{14}$ A. M. Nicolson and G. F. Ross, IEEE Trans. Instrum. Meas. 19(4), 377-382 (1970)

${ }^{15}$ W. B. Weir, Proc. IEEE 62(1), 33-36 (1974).

${ }^{16} \mathrm{~J}$. Baker-Jarvis, E. Vanzura, and W. Kissick, IEEE Trans. Microwave Theory Tech. 38, 1096-1103 (1990).

${ }^{17}$ J. Baker-Jarvis, R. G. Geyer, J. John, H. Grosvenor, M. D. Janezic, C. A. Jones, B. Riddle, and C. M. Weil, IEEE Trans. Dielectr. Electr. Insul. 5(4), 571-577 (1998)

${ }^{18}$ E. J. Vanzura, J. R. Baker-Jarvis, J. H. Grosvenor, and M. D. Janezic, IEEE Trans. Microwave Theory Tech. 42(11), 2063-2070 (1994).

${ }^{19}$ J. R. Baker-Jarvis, M. D. Janezic, J. H. Grosvenor, Jr., and R. G. Geyer, Natl. Inst. Stands. Technol. Tech. Note 1355-R, 236 (1993).

${ }^{20}$ P. Queffelec, P. Gelin, J. Gieraltowski, and J. Loaec, IEEE Trans. Magn. 30(2), 224-231 (1994).

${ }^{21}$ P. Queffelec, M. Le Floc'h, and P. Gelin, IEEE Instrum. Meas. 47(4), 956-963 (1998)

${ }^{22}$ C. M. Krowne, M. Daniel, S. W. Kirchoefer, and J. M. Pond, IEEE Trans. Microwave Theory Tech. 50(2), 537-548 (2002).

${ }^{23}$ H.-T. Lue and T.-Y. Tseng, IEEE Trans. Ultrason. Ferroelectr. Freq. Control 48(6), 1640-1647 (2001).

${ }^{24}$ M. D. Janezic, D. F. Williams, V. Blaschke, A. Karamcheti, and C. S. Chang, IEEE Trans. Microwave Theory Tech. 51(1), 132-136 (2003).

${ }^{25}$ C. L. Holloway and E. F. Kuester, IEEE Trans. Microwave Theory Tech. 43(12), 2695-2701 (1995).

${ }^{26}$ V. Laur, G. Tanne, P. Laurent, A. Rousseau, V. Bouquet, M. Guilly-Viry, and F. Huret, Ferroelectrics 353, 21 (2007). 
${ }^{27}$ S. Glinšek, B. Malič, Z. Kutnjak, H. Wang, J. Krupka, and M. Kosec, Appl. Phys. Lett. 94, 172905 (2009).

${ }^{28}$ A. Rousseau, V. Laur, S. Députier, V. Bouquet, M. Guilloux-Viry, G. Tanné, P. Laurent, F. Huret, and A. Perrin, Thin Solid Films 516, 4882-4888 (2008).

${ }^{29}$ S. Triebwasser, Phys. Rev. 114, 63 (1959).

${ }^{30}$ S. De Blasi and P. Quéffélec, European Microwave Week 2008, Amsterdam, October 2008.

${ }^{31} \mathrm{P}$. Quéffélec, M. Le Floc'h, and P. Gelin, IEEE Trans. Microwave Theory Tech. 47(4), 390-397 (1999).

${ }^{32}$ A. W. Kraszewski and S. O. Nelson, IEEE Trans. Microwave Theory Tech. 40(1), 151-155 (1992).
${ }^{33}$ C. P. L. Rubinger and L. C. Costa, Microwave Opt. Technol. Lett. 49(7), 1687-1690 (2007).

${ }^{34}$ J. M. Le Floch, F. Houndonougbo, V. Madrangeas, D. Cros, M. GuillouxViry, and W. Peng, J. Electromagn. Waves Appl. 23, 549 (2009).

${ }^{35}$ T. Lacrevaz et al., Microelectron. Eng. 83, 2184-2188 (2006).

${ }^{36}$ F. Charlet, C. Bermond, S. Putot, G. Le Carval, and B. Flechet, International Conference on Simulation of Semiconductor Processes and Devices, SISPAD, Seattle, USA (2000), p. 87.

${ }^{37}$ C. Veyres and V. F. Hanna, Int. J. Electron. 48(1), 47-56 (1980).

${ }^{38}$ A.-G. Moussavou, S. Députier, V. Bouquet, M. Guilloux-Viry, A. Perrin, R. Sauleau, and K. Mahdjoubi, Ferroelectrics 362, 137-144 (2008). 